\title{
Pleuroscopy: indications and clinical considerations
}

\author{
Stephanie K. McCarty \\ Kolene E. McDade \\ Gaetane C. Michaud
}

Thoracic Oncology Program, Yale School of Medicine, New Haven, CT, USA

\author{
Address for correspondence: \\ Gaetane C. Michaud \\ Thoracic Oncology Program \\ Yale School of Medicine \\ LCI-100C - 20 York Street \\ New Haven, Connecticut 06510, USA \\ E-mail: gaetane.michaud@yale.edu
}

\section{Summary}

Pleuroscopy is a minimally invasive procedure performed under moderate sedation that allows for direct visualization of the pleural space and therapeutic intervention. It is indicated in several clinical conditions, including diagnosis of unknown exudative pleural effusions, treatment of empyemas and pleurodesis to prevent re-accumulation of pleural effusions. It may be performed in an endoscopy suite without need for intubation, usually by a pulmonologist with training in endoscopic procedures. Pleuroscopy is generally safe and well tolerated, with few complications and very low associated mortality. It is usually considered in patients who may also be considered for VATS or tunneled pleural catheters, and often the decision of the appropriate technique is left to the clinician based on individual patient characteristics that would favor one modality over another.

KEY WORDS: pleural disease, pleuroscopy, thoracoscopy, pleural effusion, empyema, pleurodesis, VATS.

\section{Introduction}

Pleuroscopy, or medical thoracoscopy, is a percutaneous endoscopic procedure performed to directly visualize the pleural space for both diagnostic and therapeutic purposes. It is a minimally invasive alternative to video-assisted thoracoscopic surgery (VATS) and is most commonly used in the diagnosis of exudative pleural effusions of unknown etiology, as well as for pleurodesis in the management of malignant pleural effusions. It is reserved for diagnosis and management of pleural disease when less invasive methods (such as pleural fluid analysis and cytology by thoracentesis) are ineffective or more commonly, non-diagnostic. Pleuroscopy has historically achieved almost $100 \%$ diagnostic accuracy for malignant and tuberculous effusions and is generally safe and well tolerated, with a low complication rate (morbidity of $2-5 \%$ and mortality $<0.1 \%$ ). Additionally, it can be successfully used for management of recurrent or refractory malignant pleural effusions, with success rates of talc pleurodesis approaching $90 \%(1)$
Pleuroscopy is a minimally invasive alternative to VATS commonly used in the diagnosis of exudative pleural effusions of unknown etiology, as well as for pleurodesis.
Pleuroscopy was first described in a single patient report by Richard Cruise in 1866, though development of the technique is largely credited to Hans Christian Jacobeus, who began publishing on this procedure in 1910 (2). In the pre-antibiotic era, pleuroscopy was commonly performed in the treatment of empyema and tuberculous pleural effusions, but its use declined with the creation and optimization of antibiotic regimens for respiratory diseases. Pleuroscopy and VATS alike began to resurge with the success of laparoscopic abdominal procedures in the 1970 s, and by the 1990 s $5 \%$ of pulmonologists surveyed in the US stated that they performed the procedure regularly (3). It is more commonly performed in Europe, and though no data is available to support the claim, the prevalence of its use seems to be on the rise. It carries the benefit of allowing direct visualization of the pleural space with only local anesthesia and moderate sedation, and is therefore useful for several diagnostic and therapeutic indications.

\section{Indications}

\section{Diagnostic indications}

The most common indication for pleuroscopy is sampling of an exudative pleural effusion of unknown etiology, since it allows for direct visualization of the pleural space and direct biopsy of abnormal pleura. Figure 1 shows the appearance at pleuroscopy of metastatic adenocarcinoma affecting both the visceral 


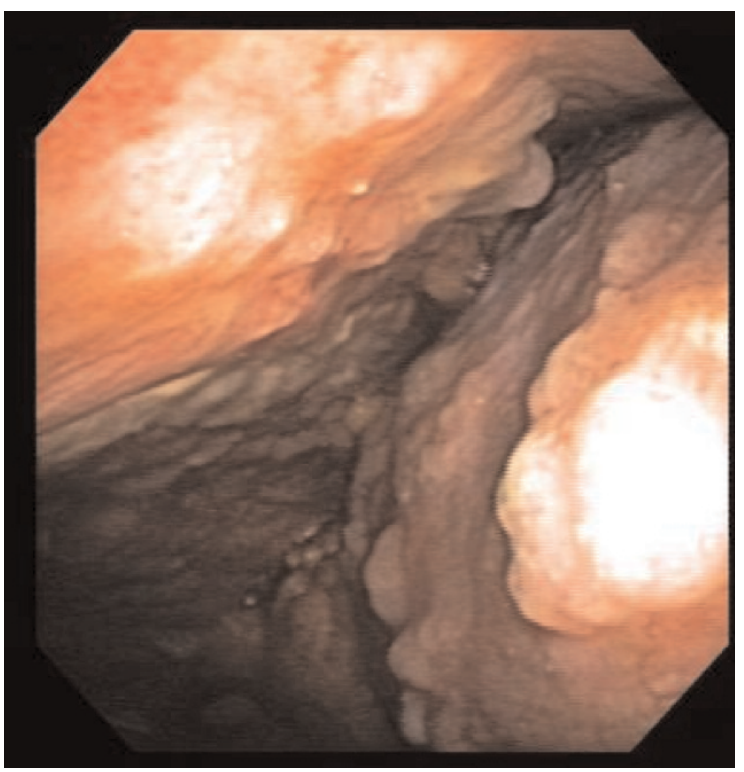

Figure 1 - Tumor infiltration of parietal and visceral pleura by metastatic adenocarcinoma of lung as seen at pleuroscopy.

and parietal pleurae. In developed countries where the incidence of tuberculosis is low, $>50 \%$ of undiagnosed pleural effusions will be found to be malignant, particularly when large or bloody (4). In the case of an unexplained pleural effusion with non-diagnostic pleural fluid analysis from thoracentesis, pleuroscopy is an absolute indication. The sen-

While simple, non-
septated empyemas
can be treated with
pleural drainage and
antibiotics alone,
pleuroscopy may be
indicated in treatment
failure or when there
are septations and lo-
culations.
sitivity of this technique has been reported in several studies to exceed that of pleural fluid analysis and closed pleural biopsy (93$97 \%$ vs $60-70 \%$ vs $40 \%$, respectively) (5-7). In addition to diagnosing malignant or infectious effusions, pleuroscopy can also be used to perform pleural based biopsies, which allow for staging of primary lung cancer or mesothelioma, as well as flow cytometry, tumor marker and genetic analysis. Figure 2 demonstrates the biopsy technique of a parietal pleural tumor implant. These additional studies may be useful in determining prognosis and optimal treatment for a given primary tumor, based on individual characteristics of the tumor. In this era of personalized medicine, the pleura is a rich source of tissue and cells for analysis and to determine targets for treatment.

The diagnosis of pleural tuberculosis can also be challenging, especially in developed countries, where the prevalence of disease is low. Sensitivity of AFB culture from sputum and thoracentesis is low, though the effusion itself is characteristically unilateral and exudative with a lymphocytic predominance. Several biomarkers have been described, particularly adenosine deaminase and interferon gamma, but the false positive

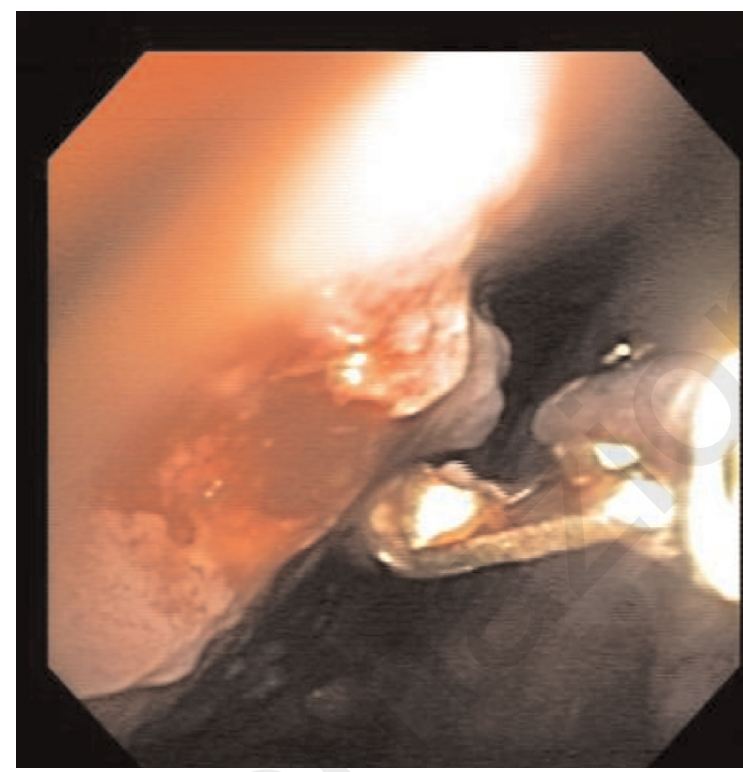

Figure 2 - Parietal pleural biopsy of pleural based metastasis by pleuroscopy.

rates in the setting of empyema and lymphomas continue to limit their usefulness (8). In many cases, diagnosis by pleuroscopy is indicated, which carries a sensitivity and specificity of $100 \%$ (9).

\section{Therapeutic indications}

Pleuroscopic pleurodesis is one of several options in the management of recurrent malignant pleural effusions that rapidly reaccumulate. The most common sclerosing agent is insufflated talc (talc poudrage), which has been shown to be moderately more successful in achieving pleurodesis compared to bleomycin and tetracycline/doxycycline. It has also been shown to be slightly more effective when insufflated at the time of thoracoscopy than as a slurry delivered through a chest tube, though this effect was more prominent in lung and breast primary tumors than others (10). Performing pleurodesis by pleuroscopy or VATS carries the added benefit of being able to pursue simultaneous diagnostic and therapeutic procedures, as well as the ability to remove adhesions, drain an existing effusion, and visualize the dispersal of talc throughout the entire pleural cavity (11). In the treatment of empyemas, pleuroscopy is an effective means by which to drain free-flowing and multiloculated collections. While simple, non-septated empyemas can be treated with thorascopic drainage and antibiotics alone, pleuroscopy may be indicated in treatment failure or when there is evidence of septations and loculations on imaging. In the Ravaglia et al. (2012) study, they found pleuroscopy plus antibiotic therapy to be effective in the treatment of $85 \%$ of all empyemas studied: $100 \%$ of free-flowing, $92 \%$ of multiloculated, and $50 \%$ of organized or multifocal empyemas (12). Pleurodesis can also be used in the treatment of recurrent pneumothorax and chylothorax. 


\section{Contraindications}

Pleuroscopy is generally a safe and well tolerated procedure, but it requires the patient to be able to tolerate conscious sedation, local anesthetic, and prolonged positioning in the lateral decubitus position while breathing spontaneously. Mortality rates are as low as $0.1 \%$. The absolute contraindications to pleuroscopy are complete obliteration of the pleural space from adhesions or pleural disease; however in some cases this can be overcome by extending the incision and/or blunt dissection of adhesions or lung tissue from the chest wall with the operator's finger. Other relative contraindications to pleuroscopy include inability to tolerate lateral decubitus positioning or induced pneumothorax, tenuous respiratory status/hypoxia, cardiopulmonary instability, bleeding diathesis or coagulopathy, or allergy to any of the necessary medications.

\section{Procedure}

Pre-procedural preparations \& considerations

As with any other procedure, a detailed history and physical examination of the patient is the first step in assessing the safety and appropriate indication for pleuroscopy. Particular attention should be paid to un-
Particular attention should be paid to underlying pulmonary disease, medical comorbidities, use of anticoagulants or steroids, prior radiation or occupational exposures, and drug intolerance. derlying pulmonary disease, medical comorbidities, use of anticoagulants or steroids, prior radiation or occupational exposures, and drug intolerances. The physical exam should be supplemented with chest radiography, CT scan, and bedside ultrasound for determination of the optimal site of insertion of the thoracoscope, as well as basic evaluation of the patient's cardiac, pulmonary, liver, and kidney function through pulse oximetry, EKG, and lab work. A CBC and type and screen should also be done in the event of need for transfusion. Informed consent should be obtained in the standard practice, with explanation of the rationale for procedure, risks, benefits, and alternatives, common complications, and expected result of the procedure. The endoscopy suite should be prepared with trained personnel and all necessary equipment.

\section{Procedural technique}

Pleuroscopy is a sterile procedure. The patient is placed in the lateral decubitus position with the affected side up for the duration of the procedure, and has cardiac, blood pressure, and oxygen saturation monitoring throughout. Ultrasound is used to confirm the location of the hemidiaphragm, as well as to identify any loculations or pleural thickening that may cause complications. A common entry site is mid or anterior axillary line in the fifth intercostal space.

When the patient is in position and the operators scrubbed, the patient is sedated, usually with midazolam and fentanyl. The entry site is numbed with lidocaine from the skin, through the intercostals, and extending to the pleural space. Once numb, a large bore needle is inserted into the thorax and a small pneumothorax is induced. A larger incision is made to allow for blunt dissection and insertion of the trocar into the thoracic space, with care being taken to avoid trauma to the lung and intercostal vessels. The pleuroscope is placed through the trocar and into the thoracic space. Once in the pleural space, the pleuroscope is used for complete visualization of the thoracic cavity. A second trocar may be introduced under direct visualization to allow for biopsy, forceps, or tubing to introduce insufflated talc. Biopsy specimens, fluid samples, and cultures can be obtained, and any therapeutic intervention (insufflation of talc for pleurodesis, for example) can be performed.

When all diagnostic and therapeutic procedures are complete, the pleuroscope and trocars are removed. A chest tube is inserted for temporary drainage and sutured into place. The patient is typically monitored for a brief period of time while they are recovering from conscious sedation, but it is not necessary for them to be admitted to the hospital for further monitoring unless complications arise.

\section{Complications}

Overall, pleuroscopy is a safe and well tolerated procedure, especially in the hands of an experienced operator. The British Thoracic Society in 2010 pooled data from 47 studies to establish guidelines for use and caution against documented complications. This analysis demonstrated an overall major complication rate of $1.8 \%$, with major complications defined as empyema, hemorrhage, port site tumor invasion, bronchopleural fistula, post-operative pneumothorax/ prolonged air leak, and pneumonia. Minor complications - subcutaneous emphysema, minor hemorrhage, skin infection, periprocedure hypotension, fever, or atrial fibrillation were reported in $7.3 \%$ of cases (13). Other potential complications include peri-procedural injury to the lung tissue, re-expansion pulmonary edema, or seeding of the chest wall by tumor cells (14). Patients should be counseled to expect some degree of post-procedural pain (especially if pleurodesis was pursued), and practitioners should be prepared to address this expected outcome. 


\section{Clinical practice}

The utility of pleuroscopy in numerous conditions has been described in earlier sections: near $100 \%$ diagnostic accuracy of both malignant and tuberculous pleural effusions, as well as successful treatment of recurrent malignant pleural effusions and empyemas. Further discussion will focus on the comparison of this
Pleurodesis by talc poudrage is effective in preventing re-accumulation of malignant pleural effusions in $75-87 \%$ of patients, with corresponding improvement in subjective dyspnea. technique to VATS and tunneled pleural catheters, since the clinical decision of optimal therapy between these three techniques, particularly in the management of malignant pleural effusions, is often encountered.

\section{Pleuroscopy vs VATS}

Pleuroscopy and VATS are two different techniques that allow for direct visualization of the pleural space and can be used for sometimes overlapping indications, especially in the diagnosis and management of malignant pleural effusions and empyemas. While pleuroscopy is performed in an endoscopy suite under local anesthesia and moderate sedation, VATS is performed in an operating room with general anesthesia, intubation and single lung ventilation.

VATS has a broader diagnostic and therapeutic scope than does pleuroscopy, but carries a higher complication rate due to the need for general anesthesia and intubation as well as the complexity of the disease entities being addressed. It can be used in multi-loculated or organized effusions and empyemas, as well as for decortication of lung tissue, wedge lung biopsy, lung nodule resection, lobectomy, and esophageal procedures (15). Both pleuroscopy and VATS can be useful in cancer staging, since metastases to the pleura defines the primary malignancy as stage IV, and therefore inoperable. In this case, pleuroscopy may be the preferred technique since it allows for staging of the primary tumor with potential for delivering talc poudrage in the same session, potentially sparing the patient a VATS and more extensive intervention if indeed there is evidence of pleural spread (16).

Pleuroscopy is also the procedure of choice in the diagnosis of mesothelioma, since the biopsies obtained through either method are comparable, and it is the less invasive approach of the two (17). Pleuroscopy and VATS should be utilized in a continuum of care in the management of empyema. As shown in Ravaglia et al. (2012), pleuroscopic drainage of an empyema in combination with antibiotics is curative in $92-100 \%$ of cases. However, when there is evidence of pleural thickening, extensive septations, or organization of the empyema, VATS is required for more extensive drainage and decortication than is possible by pleuroscopy (12).

In general, patients who are able to tolerate sponta- neous breathing with localized and non-complicated pleural disease should undergo pleuroscopy, as it is less invasive and carries less risk than does VATS. VATS, however, may be the procedure of choice in patients who are unstable or who have more complicated pleural and pulmonary disease.

\section{Pleuroscopy vs tunneled pleural catheters in the management of malignant pleural effusions}

Tunneled pleural catheters were approved by the FDA in 1997 for management of malignant pleural effusions, and there has since been great debate over the optimal long-term treatment of these effusions. Pleurodesis offers definitive management of the production of pleural fluid; tunneled pleural catheters drain the accumulated fluid, and in some cases promote spontaneous pleurodesis allowing for removal of the catheter. Particular challenges that arise when considering therapy for malignant pleural effusions are (1) the rapid re-accumulation of pleural effusions if not managed definitively and (2) the adverse effect profile and length of hospital stay in these patients, since median survival in patients with a malignant pleural effusion typically ranges between 4-9 months, and quality of life is paramount (18).

Pleurodesis by talc poudrage is effective in preventing re-accumulation of malignant pleural effusions in 75$87 \%$ of patients, with corresponding improvement in subjective dyspnea (19). Tunneled pleural catheters, on the other hand, are effective in relieving dyspnea in roughly $90 \%$ of patients by facilitating drainage of the effusion. They also achieve spontaneous pleurodesis with resulting decrease in effusion re-accumulation in $40-50 \%$ of patients within 2 months (20). Numerous studies have demonstrated that the two procedures offer comparable relief of symptoms with no significant difference in complication rates, but that tunneled pleural catheters have a more favorable post-procedure profile compared to pleurodesis, with shorter overall and post-procedure length of stay, fewer re-interventions on the ipsilateral side, and lower cost (2123). Despite these results, the decision of pleurodesis versus tunneled pleural catheter continues to be a discussion that is multi-disciplinary including the patient, their goals of care, and expected survival, and the physician's clinical impression.

In addition to ongoing head to head studies, Reddy et al. (2011) conducted a pilot study investigating the safety and effectiveness of performing simultaneous pleurodesis and tunneled pleural catheter insertion with promising initial results. They demonstrated in a small patient population (30 patients) that combination therapy could produce equivalent symptom control with a comparable safety profile, rate of pleurodesis, and hospital length of stay to either therapy alone, with the added benefit of removal of the tunneled pleural catheter one week after placement (24). Larger studies are necessary to validate these results, but represent a promising future direction in the management of malignant pleural effusions. 


\section{Conclusion}

Pleuroscopy is a safe and effective therapy in the diagnosis and treatment of pleural disease. Use of this technique should always be considered in patients with exudative pleural effusions of unknown etiology, and discussed as a minimally invasive alternative to VATS when safe to do so. The clinical decision of pleurodesis versus tunneled pleural catheter in the management of malignant pleural effusions should be discussed with the patient and tailored to their goals of care as an individual, since both techniques offer symptomatic relief with similar side effect profiles. Research is underway to compare outcomes and side effect profiles between these two therapies, and in combination with each other, to better guide therapy decisions in the future.

\section{References}

1. Michaud G, Berkowitz D, Ernst A. Pleuroscopy for diagnosis and therapy for pleural effusions. Chest. 2010;138(5):1242-1246.

2. Moisiuc FV, Colt HG. Thoracoscopy: origins revisited. Respiration. 2007;74:344-355.

3. Loddenkemper R. Medical Thoracoscopy/Pleuroscopy. Principles and Practice of Interventional Pulmonology. 2013;605-621.

4. Froudarakis M. Diagnostic thoracoscopy: malignant pleural effusion. Thoracoscopy for Pulmonologists. 2014;87-104.

5. Loddenkemper R. Thoracoscopy - state of the art European Respiratory Journal. 1998;111:213-221.

6. Blanc FX, Atassi K, Bignon J, Housset B. Diagnostic value of medical thoracoscopy in pleural disease: a 6-year retrospective study. Chest. 2002;1215:16771683.

7. Prabhu VG, Narasimhan R. The role of pleuroscopy in undiagnosed exudative pleural effusion. Lung India. 2012;29(2):128-130.

8. Bays AM, Pierson DJ. Tuberculous pleural effusion. Respiratory Care. 2012;57(10):1682-1684.

9. Diacon AH, Van de Wal BW, Wyser C, et al. Diagnostic tools in tuberculous pleurisy: a direct comparative study. European Respiratory Journal. 2003;224:589591.

10. Dresler CM, Olak J, Herndon 2nd JE, et al. Phase 3 intergroup study of talc poudrage vs talc slurry sclerosis for malignant pleural effusion. Chest. 2005;
127:909-915.

11. Hsia D, Musani Al. Management of malignant pleural effusions. Current Respiratory Care Reports. 2012;1(2):73-81.

12. Ravaglia $C$, Gurioli $C$, Tomassetti $S$, et al. Is medical thoracoscopy efficient in the management of multiloculated and organized thoracic empyema? Respiration. 2012;84:219-224.

13. Rahman NM, Ali NJ, Brown G, et al. Local anaesthetic thoracoscopy: British Thoracic Society pleural disease guideline 2010. Thorax. 2010;65:54-60.

14. Loddenkemper R, Lee P, Noppen M, Mathur PN. Medical thoracoscopy/pleuroscopy: step by step. Breathe. 2011;8(2):156-167.

15. Lee $P$, Colt HG. Pleuroscopy in 2013. Clinics in Chest Medicine. 2013;34(1):81-91.

16. American Thoracic Society. Management of malignant pleural effusions. American Journal of Respiratory and Critical Care Medicine. 2000;1987-2001.

17. Hollevoet K, Nackaerts K, Thimpont J, et al. Diagnostic performance of soluble mesothelin and megakaryocyte potentiating factor in mesothelioma. American Journal of Respiratory and Critical Care Medicine. 2010;620-625

18. Bielsa S, Martin-Juan J, Porcel JM, et al. Diagnostic and prognostic implications of pleural adhesions in malignant effusions. Journal of Thoracic Oncology. 2008;3:1251-1256.

19. Aelony Y, King RR, Boutin C. Thoracoscopic talc poudrage in malignant pleural effusions: effective pleurodesis despite low pH. Chest. 1998;113:1007-1012.

20. Suzuki K, Servais EL, Rizk, NP, et al. Palliation and pleurodesis in malignant pleural effusion: the role for tunneled pleural catheters. Journal of Thoracic Oncology. 2011;6(4):762-767.

21. Hunt BM, Farivar AS, Vallieres E, et al. Thoracoscopic talc versus tunneled pleural catheters for palliation of malignant pleural effusions. The Annals of Thoracic Surgery. 2012;94(4):1053-1059.

22. Fysh ETH, Waterer GW, Kendall PA, et al. Indwelling pleural catheters reduce inpatient days over pleurodesis for malignant pleural effusion. Chest. 2012;142(2):394-400.

23. Puri V, Pyrdeck TL, Crabtree TD, et al. Treatment of malignant pleural effusion: a cost-effectiveness analysis. The Annals of Thoracic Surgery. 2012;94(2):374380.

24. Reddy C, Ernst A, Lamb C, et al. Rapid pleurodesis for malignant pleural effusions: a pilot study. Chest. 2011;139(6):1419-1423. 